\title{
Heparina de baixo peso molecular no tratamento da tromboembolia pulmonar*
}

\begin{abstract}
Renato Maciel ${ }^{1}$
Este artigo de revisão analisa as propriedades farmacocinéticas das heparinas de baixo peso molecular, compara os resultados do seu uso com heparina não fracionada no tratamento da tromboembolia pulmonar, mostrando que são uma alternativa segura e efetiva. Sugere que as heparinas de baixo peso molecular administradas por via subcutânea uma ou duas vezes ao dia e sem necessidade de monitorização laboratorial freqüente permitem o manejo ambulatorial ou com internação hospitalar breve de alguns casos de tromboembolia pulmonar, resultando em redução de custos e maior satisfação do paciente. (J Pneumol 2002;28(3):137-142)
\end{abstract}

Low-molecular-weight heparin in the treatment of pulmonary embolism

This article reviews the pharmacokinetic properties of low-molecular weight heparins, compares the results of their use to that of unfractioned heparin in a pulmonary embolism treatment, showing they are a safe an effective alternative. The authors suggest that low-molecular-weight heparins administered subcutaneously once or twice-daily and without frequent laboratory monitoring have permitted out-of-hospital or short hospitalization management in some cases of pulmonary embolism, resulting in cost savings and increased patient satisfaction.

Descritores - Heparina. Heparina de baixo peso molecular. Embolia pulmonar.

Key words - Pulmonary embolism. Heparin. Low-molecular-weight heparin.

\section{INTRODUÇÃO}

Trombose venosa profunda (TVP) e tromboembolia pulmonar (TEP) são causas comuns de morbidade e mortalidade e constituem as manifestações clínicas da tromboembolia venosa (TEV). Recentemente, Kenneth Moser se referiu a essa ocorrência como substancial e inaceitável(1).

Vários estudos têm pesquisado a freqüência de TVP e TEP.

No estudo de Worcester (2), conduzido em 16 hospitais dessa região metropolitana, a incidência anual de TVP foi

* Trabalho realizado na Faculdade de Ciências Médicas de Minas Gerais - Belo Horizonte.

1. Professor Assistente de Pneumologia.

Endereço para correspondência - Rua Tomé de Souza, 300, apto. 1.202 - 30140-130 - Belo Horizonte, MG. Tel. (31) 3223-0484; fax (31) 3241-2220. E-mail: renatomaciel@ task.com.br

Recebido para publicação em 21/11/01. Aprovado, após revisão, em 17/1/02.
Siglas e abreviaturas utilizadas neste trabalho

AT III - Antitrombina III

EV - Endovenosa

HBPM - Heparina de baixo peso molecular

HNF - Heparina não fracionada

RNI - Razão internacional de normalização

TEP - Tromboembolia pulmonar

TEV - Tromboembolia venosa

TIH - Trombocitopenia induzida pela heparina

TTPa - Tempo de tromboplastina parcial ativado

TVP - Trombose venosa profunda

UCh - Unidade Choay

UI - Unidade internacional

UTI - Unidade de tratamento intensivo

de 48 por 100.000 e de TEP com ou sem TVP, de 23 por 100.000 , com maior freqüência no sexo masculino e em pacientes idosos.

Stein et al. ${ }^{\left({ }^{3}\right)}$ pesquisaram a ocorrência de TEP diagnosticada em 175.730 internações em hospital geral e encontraram 400 casos $(0,23 \%)$.

Dismuke e Wagner (4) pesquisaram TEP como causa de morte em um hospital universitário no período de 1966 a 1980. Foram realizadas 3.412 necropsias, em um total de 187.524 internações. Considerou-se TEP como principal causa de morte os casos que apresentaram sinais recentes de oclusão vascular superior a $50 \%$ do leito arte- 
rial pulmonar. Foram encontrados 203 casos de TEP maciço (6\% das mortes).

Lindblad et al. ${ }^{(5)}$, revendo a ocorrência de embolia pulmonar fatal em 994 necropsias realizadas em hospital geral na Suécia, encontraram 93 casos $(9,4 \%)$.

No Brasil, Maffei et al.(6), em trabalho de revisão de 998 necropsias realizadas na Faculdade de Medicina de Botucatu, no período de 1969 a 1976, encontraram 166 ocorrências de embolia pulmonar, das quais 38 tiveram TEP como causa de morte devido a sua extensão ou gravidade.

Mais recentemente, Menna Barreto et al. ${ }^{(7)}$ revisaram os laudos de necropsias realizadas no Serviço de Patologia do Hospital de Clínicas de Porto Alegre entre janeiro de 1985 e dezembro de 1995. Nas 767 necropsias foram encontrados 30 casos de TEP $(3,9 \%)$, sendo que em três casos a tromboembolia pulmonar foi considerada a causa principal de morte.

\section{HePARINA E hePARINA dE BAIXO PESO MOLECULAR}

Heparina não fracionada (HNF) é uma mistura heterogênea de moléculas compostas por cadeias de polissacárides com peso molecular variando de 3.000 a 30.000 dáltons, média de 15.000 dáltons, correspondendo a 45 sacárides.

Heparinas de baixo peso molecular (HBPM) são fragmentos de HNF obtidos por despolimerização química ou enzimática com peso molecular variando de 1.000 a 10.000 dáltons, média de cerca de 5.000 dáltons(8).

HNF e HBPM exercem a sua ação anticoagulante através da ativação da antitrombina III, que tem a sua atividade acelerada em até 1.000 vezes no sentido de inibir os fatores da coagulação lla e $X a$ e, em menor proporção, IXa, Xla e XIla. A ligação da HNF e HBPM à antitrombina III depende da presença de uma única seqüência de pentassacárides contida em cerca de um terço das moléculas de heparina. Os dois terços restantes têm mínima atividade anticoagulante nas concentrações terapêuticas usuais. Esta seqüência de pentassacárides confere alta afinidade da HNF e HBPM pela antitrombina III(8-10). Qualquer molécula de heparina ou HBPM que contenha o pentassacáride pode inibir a ação $X a$ simplesmente pela ativação da AT III. Para inativar a trombina (Ila), a HNF ou HBPM tem que se ligar à antitrombina III e ao fator Ila simultaneamente, formando um complexo ternário, que só ocorre com cadeias mais longas, com pelo menos 18 sacárides $^{(11)}$, conforme a Figura 1.

Atualmente, um pentassacáride sintético, com atividade exclusivamente anti-Xa está em fase de avaliação clínica para prevenção e tratamento de TEV.

Além da redução do efeito anti-lla, a HBPM apresenta outras vantagens farmacocinéticas em relação à $\mathrm{HNF}$ :

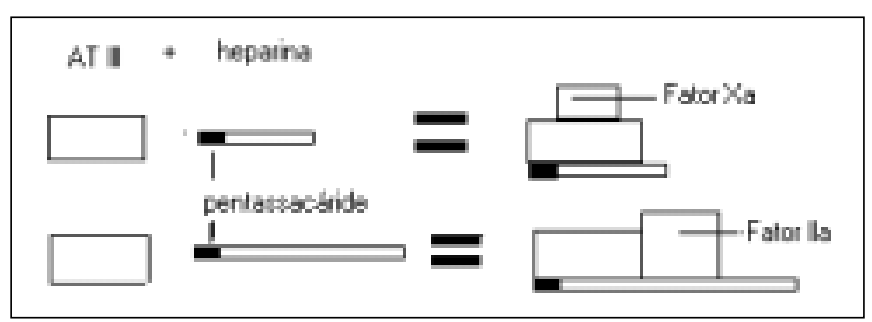

Figura 1 - A inibição do fator Xa pela antitrombina III requer apenas a presença do pentassacáride na molécula de heparina, enquanto a inibição do fator lla requer a formação de um complexo ternário (heparina - AT3-I/a), que implica a presença de moléculas maiores de heparina com pelo menos 18 sacárides

1) Menor ligação a proteínas plasmáticas e proteínas de fase aguda.

2) Menor ligação aos macrófagos e células endoteliais.

3) Menor ligação às plaquetas e fator plaquetário 4 (PF4).

4) Menor ligação aos osteoblastos.

Tais diferenças proporcionam vantagens terapêuticas(812), como resposta anticoagulante mais previsível, meiavida plasmática mais longa, maior biodisponibilidade, redução da trombocitopenia induzida pela heparina - $\mathrm{TIH}^{(13)}$ e menor osteopenia. Estas propriedades permitem que as HBPM possam ser usadas uma ou duas vezes ao dia por via subcutânea, sem necessidade de linha venosa e bomba de infusão e com monitorização laboratorial mínima(14).

As heparinas de baixo peso molecular e algumas de suas características estão relacionadas na Tabela 1.

\section{TRATAMENTO DA TROMBOEMBOLIA PULMONAR}

O tratamento clássico de TEP consiste na administração inicial de heparina não fracionada EV em bolus de $80 \mathrm{Ul} / \mathrm{kg}$, seguida de infusão contínua na dose de 18UI/ $\mathrm{kg}$ por hora(15), dose ajustada para manter o TTPa entre 1,5 e 2,3 vezes o valor de controle. Warfarina deve ser iniciada nas primeiras 24 horas, $5 \mathrm{mg}$ por dia, sem dose de ataque ${ }^{(16)}$. 0 tratamento simultâneo com os dois medicamentos será mantido pelo menos por cinco dias, e heparina será suspensa após RNI acima de 2,0 por dois dias consecutivos $(8,12,17)$.

TABELA 1

Heparinas de baixo peso molecular disponíveis atualmente no Brasil

\begin{tabular}{clccc}
\hline \multicolumn{1}{c}{ Droga } & $\begin{array}{c}\text { Nome } \\
\text { comercial }\end{array}$ & $\begin{array}{c}\text { Peso } \\
\text { molecular }\end{array}$ & Meia-vida & Anti-Xa/lla \\
\hline Dalteparina & Fragmin & 6.000 dáltons & $2,7 \mathrm{~h}$ & $2,7: 1,0$ \\
Enoxaparina & Clexane & 4.200 dáltons & $4,5 \mathrm{~h}$ & $3,8: 1,0$ \\
Nadroparina & Fraxiparina & 4.500 dáltons & $2,7 \mathrm{~h}$ & $3,6: 1,0$ \\
\hline
\end{tabular}


TABELA 2

Eficácia e segurança da heparina de baixo peso molecular em comparação com heparina não fracionada no tratamento do TEV, em dois estudos

\begin{tabular}{llrrrrr}
\hline & Tratamento & N & Recorr. TEV & Recorr. TEP & Sangr. maior & Morte/TEP \\
\hline \multirow{2}{*}{ Columbus $^{(20)}$} & HNF & 511 & $25(4,9 \%)$ & $12(2,4 \%)$ & $8(1,6 \%)$ & $3(0,6 \%)$ \\
& HBPM & 510 & $27(5,3 \%)$ & $10(2,0 \%)$ & $10(2,0 \%)$ & $3(0,6 \%)$ \\
\hline Simonneau et al.(21) & HNF & 308 & $6(1,9 \%)$ & $4(1,3 \%)$ & $5(1,6 \%)$ & $3(1,0 \%)$ \\
& HBPM & 304 & $5(1,6 \%)$ & $4(1,3 \%)$ & $3(1,0 \%)$ & $3(1,0 \%)$ \\
\hline
\end{tabular}

\section{HePARINA de BAIXo PESO MOLECUlar NO TRATAMENTO DE TEP}

Em 1989, Albada et al. ${ }^{(18)}$ apresentaram um trabalho prospectivo, randomizado, duplo-cego, comparando o tratamento de TEV com HNF ou HBPM (dalteparina) em 194 pacientes, dos quais 70 tinham o diagnóstico de TEP. Todos foram tratados por via endovenosa em infusão contínua e com monitorização laboratorial para manter os níveis séricos de ação anti-Xa entre 0,3 e 0,9 unidades por ml. A conclusão dos autores foi de que heparina de baixo peso molecular EV dose ajustada é tão segura e efetiva quanto HNF no tratamento de TEV agudo. Deve-se ressaltar que ali não foram ainda utilizadas as vantagens farmacocinéticas das HBPM.

A primeira publicação dirigida exclusivamente ao tratamento de TEP com heparina de baixo peso molecular foi de Thery et al.(19), que compararam HNF com três regimes terapêuticos de Fraxiparina: 400, 600 e 900 unidades Choay anti-Xa/kg de peso por dia, dose fixa, no tratamento de embolia pulmonar submaciça (1U Choay = $0,38 \mathrm{UI}$ anti-Xa).

Trata-se de um estudo multicêntrico, prospectivo, randomizado, envolvendo 101 pacientes. Fraxiparina foi administrada por via subcutânea, duas ou três vezes ao dia, sem controle laboratorial. Os grupos que receberam 900 $(n=7)$ e $600(n=26) \mathrm{UCh} / \mathrm{kg} / \mathrm{dia}$ apresentaram taxas elevadas de sangramento, respectivamente, $57 \%$ e $19 \%$, tendo sido interrompidos. 0 terceiro regime terapêutico, $400 \mathrm{UCh} / \mathrm{kg} / \mathrm{dia}(\mathrm{n}=35)$, foi tão efetivo e seguro quanto o tratamento com HNF endovenosa $(n=33)$, com bolus inicial de $50 \mathrm{Ul} / \mathrm{kg}$ seguido de infusão contínua, dose ajustada, para manter o TTPa entre 2,5 e 3,0 vezes o controle.

Em 1997 foram publicadas duas grandes séries comparando HNF com HBPM no tratamento da tromboembolia venosa.

No estudo Columbus(20), 1.021 pacientes com diagnóstico de TVP ou TEP foram randomizados para uso de Reviparina subcutânea, duas vezes ao dia, dose fixa de acordo com o peso corporal, ou heparina EV, dose ajustada para
TABELA 3

Redução da obstrução vascular na cintilografia pulmonar de perfusão após sete dias de tratamento do TEP com HBPM ou HNF(21)

\begin{tabular}{lllll}
\hline & $\begin{array}{c}\text { Obstrução } \\
\text { vascular inicial }\end{array}$ & $\begin{array}{c}\text { Redução da } \\
\text { obstrução vascular }\end{array}$ \\
\hline HNF & $\mathrm{n}=274$ & $46 \%$ & $\mathrm{n}=260$ & $19 \%$ \\
HBPM & $\mathrm{n}=273$ & $47 \%$ & $\mathrm{n}=258$ & $18,4 \%$ \\
\hline
\end{tabular}

manter TTPa 1,5 a 2,5 vezes o controle, após bolus de 5.000UI. No grupo HNF $(n=511)$, havia 378 TVP e 133 TEP e, no HBPM ( $n=510), 372$ TVP e 138 TEP. Anticoagulante oral foi administrado no primeiro ou segundo dia e mantido por 12 semanas com RNI entre 2,0 e 3,0.

No artigo de Simonneau et al.(21), 612 pacientes com diagnóstico de TEP agudo sintomático sem indicação de trombolíticos foram randomizados para terapêutica com Tinzaparina, $175 \mathrm{UI} / \mathrm{kg} / \mathrm{dia}$, dose única diária subcutânea ( $n=304$ ), ou heparina EV, com bolus inicial de $50 \mathrm{Ul} / \mathrm{kg}$, seguida de infusão contínua com dose ajustada para manter 0 TTPa entre duas a três vezes o controle $(n=308)$. Terapia com anticoagulante oral foi iniciada entre o primeiro e terceiro dia e mantida por três meses com RNI entre 2,0 e 3,0.

Os resultados destes estudos são apresentados na Tabela 2 .

No estudo de Simonneau et al.(21) foi feita também uma comparação da resposta ao tratamento com cintilografias pulmonares de perfusão realizadas nos dias 1 e 8 , com resultados apresentados na Tabela 3.

Observados em conjunto, esses dois resultados apontam para a eficácia e segurança das HBPM comparativamente à HNF.

Uma metanálise ${ }^{(22)}$ selecionou 13 estudos randomizados, controlados, publicados até 1997, que compararam HNF com HBPM na terapia da TEV. Foram incluídos 883 pacientes ( $20 \%$ do total) com diagnóstico de embolia pulmonar. Não houve diferença estatisticamente significati- 
va em relação à taxa de recorrência, sangramento maior ou menor e trombocitopenia.

0 risco de mortalidade total favoreceu HBPM - RR = $0,76[95 \% \mathrm{Cl}-0,590,98]$, porém não houve redução de TEP fatal. Uma possível hipótese para explicar essa diferença, segundo os autores, seria um efeito antineoplásico das HBPM. Tal propriedade também foi sugerida por Prandoni em outra publicação(23).

Nessa metanálise a comparação de HBPM administrada uma ou duas vezes ao dia não interferiu nos resultados finais.

Duas novas grandes séries compararam HNF com HBPM no tratamento da TEV.

Hull et al.(24), em um estudo multicêntrico, duplo-cego, randomizado, compararam Tinzaparina, dose fixa de $175 \mathrm{Ul} / \mathrm{kg}$ subcutânea uma vez ao dia $(\mathrm{n}=97)$, com HNF, bolus inicial de 5.000Ul, seguida de infusão endovenosa contínua, dose ajustada para manter o TTPa entre 1,5 e 2,5 vezes o valor controle ( $n=103$ ), no tratamento de pacientes portadores de TEP com TVP associada, com três meses de seguimento. Os resultados estão na Tabela 4.

Dos sete casos de recorrência nos pacientes em tratamento com HNF houve quatro TEP e três TVP, com diferença estatisticamente significativa a favor de HBPM ( $p=$ 0,009).

Merli et al.(25) apresentaram estudo multicêntrico, randomizado, controlado, parcialmente cego (HBPM, uma ou duas vezes ao dia), comparando enoxaparina, dose fixa, subcutânea, $1,0 \mathrm{mg} / \mathrm{kg}$ duas vezes ou $1,5 \mathrm{mg} / \mathrm{kg}$ uma vez ao dia, ou HNF com bolus inicial e infusão EV contínua, dose ajustada para manter o TTPa entre 55 e 80 segundos, seguido de anticoagulante oral, no tratamento de 900 pacientes sintomáticos com TEP $(n=287)$ ou TVP, com três meses de acompanhamento. Os resultados estão na Tabela 5 .

A conclusão dos autores sugere que enoxaparina subcutânea $1 \mathrm{mg} / \mathrm{kg}$ duas vezes ou $1,5 \mathrm{mg} / \mathrm{kg}$ uma vez ao dia é tão segura e efetiva quanto heparina EV por infusão contínua no tratamento da tromboembolia venosa.

$\mathrm{Na}$ análise de subgrupos de portadores de neoplasia ou obesidade, notou-se tendência, sem significação estatísti$\mathrm{ca}$, de taxas mais elevadas de recorrência de TEV em pa-

\section{TABELA 4}

Resultados comparativos do tratamento com HBPM ou HNF de pacientes portadores de TEP e TVP associada

\begin{tabular}{lcl}
\hline \multicolumn{1}{c}{ Eventos } & HNF $(\mathrm{n}=103)$ & HBPM $(\mathrm{n}=97)$ \\
\hline Recorrência de TEV & $7(6,8 \%)$ & 0 \\
Sangramento maior & $2(1,9 \%)$ & $1(1,0 \%)$ \\
Sangramento menor & $3(2,9 \%)$ & $1(1,0 \%)$ \\
Morte por TEP & $1(1,0 \%)$ & 0 \\
\hline
\end{tabular}

cientes em uso de enoxaparina uma vez em comparação com duas vezes ao dia.

Todas essas publicações têm evidenciado que HBPM administrada por via subcutânea, dose fixa de acordo com o peso corporal, uma ou duas vezes ao dia, é não menos e, provavelmente, mais efetiva e segura que HNF no tratamento inicial da tromboembolia pulmonar sem instabilidade hemodinâmica.

\section{TRATAMENTO DOMICILIAR DA TEP?}

Dois artigos publicados em $1996^{(26,27)}$ evidenciaram que pacientes selecionados, com diagnóstico de TVP proximal, poderiam ser medicados no domicílio com HBPM seguida de warfarin. No estudo Columbus ${ }^{(20)}$ também foram relatados casos tratados com permanência hospitalar curta e complementação do tratamento em casa com HBPM, sob supervisão do serviço de enfermagem.

O tratamento da TVP no domicílio ou com hospitalização mais breve gera redução de custos e melhora da qualidade de vida(17,26,27). Podemos, então, imaginar se tais achados poderiam ser extrapolados para portadores de TEP.

Wells et al. (28) relataram o tratamento domiciliar de 194 pacientes com diagnóstico de TVP proximal $(n=154)$, TVP de membro superior $(n=6)$ OU TEP $(n=34)$, com dalteparina subcutânea, $100 \mathrm{Ul} / \mathrm{kg}$ duas vezes $(n=38)$ ou $150 \mathrm{Ul} / \mathrm{kg}$ uma vez ao dia $(n=156)$, seguido de warfarin iniciado nas primeiras 24 horas. Foram excluídos do protocolo pacientes com co-morbidades que justificavam internação, sinais de sangramento ativo, instabilidade hemodinâmica, dor requerendo analgésicos endovenosos, idade inferior a 18 anos, hipoxemia com necessidade de oxigenioterapia e aqueles provavelmente não cooperatiVOS. HBPM domiciliar foi administrada por enfermeira (n = 95) ou pelo próprio paciente ou responsável adequadamente treinado $(n=99)$.

Todos receberam um número telefônico de urgência que podia ser acionado 24 horas por dia e eram contactados diariamente por uma enfermeira durante os três

TABELA 5

Segurança e eficácia de dois esquemas de tratamento do TEV com HBPM em comparação com heparina não fracionada

\begin{tabular}{lrrr}
\hline \multicolumn{1}{c}{ Eventos } & $\begin{array}{c}\text { HNF } \\
(\mathrm{n}=290)\end{array}$ & $\begin{array}{r}\text { Enox. 1X } \\
(\mathrm{n}=298)\end{array}$ & $\begin{array}{r}\text { Enox. 2X } \\
(\mathrm{n}=312)\end{array}$ \\
\hline Recorrência de TEV & $12(4,1 \%)$ & $13(4,4 \%)$ & $9(2,9 \%)$ \\
Recorrência de TEP & $4(1,4 \%)$ & $2(0,7 \%)$ & $2(0,6 \%)$ \\
M ortes por TEP & $2(0,7 \%)$ & $1(0,3 \%)$ & $2(0,6 \%)$ \\
Sangramento maior & $6(2,1 \%)$ & $5(1,7 \%)$ & $4(1,3 \%)$ \\
\hline
\end{tabular}


TABELA 6

Segurança e eficácia do tratamento domiciliar do TEV com HBPM

\begin{tabular}{lccc}
\hline \multicolumn{1}{c}{ Evento } & $\begin{array}{c}\text { Adm. } \mathrm{p} / \text { enferm. } \\
(\mathrm{n}=95)\end{array}$ & $\begin{array}{c}\text { Autoadm. } \\
(\mathrm{n}=99)\end{array}$ & $\begin{array}{c}\text { Total } \\
(\mathrm{n}=194)\end{array}$ \\
\hline Recorrência de TEV & $3(3,2 \%)$ & $4(4,0 \%)$ & $7(3,6 \%)$ \\
Sangramento maior & $2(2,1 \%)$ & $2(2,0 \%)$ & $4(2,0 \%)$ \\
Morte por TEP & 0 & 0 & 0 \\
\hline
\end{tabular}

meses de duração do estudo. Os resultados estão na Tabela 6 .

Não houve diferença significativa na freqüência dos desfechos acima em relação aos achados de literatura. A conclusão dos autores sugere que educação e acompanhamento cuidadoso, com pronto acesso aos profissionais de saúde em casos de urgência, tornam não inadequada a terapia domiciliar da TEP sem instabilidade hemodinâmica com HBPM.

Recentemente, o mesmo grupo de autores ${ }^{(29)}$ publicou um estudo prospectivo reunindo 158 pacientes com diagnóstico de embolia pulmonar tratados com dalteparina $200 \mathrm{Ul} / \mathrm{kg}$ subcutânea, dose única diária. Destes, 50 foram tratados internados, 27 tiveram hospitalização curta, um a três dias, e 81 receberam tratamento exclusivamente ambulatorial. Critérios de internação incluíram instabilidade hemodinâmica, hipóxia, dor intensa com uso de analgésico parenteral, risco de sangramento e co-morbidades. Nos 108 casos de tratamento externo, aconteceram seis recorrências de TEV, dois sangramentos maiores e quatro mortes (nenhuma por TEP ou sangramento). Os autores concluem que 0 tratamento ambulatorial, com HBPM, de pacientes selecionados com diagnóstico de embolia pulmonar é factível e seguro.

\section{RESTRIÇÕES AO USO DE HBPM}

Algumas restrições devem ser feitas ao uso de HBPM no tratamento da TEP:

1) Não existe evidência na literatura para tratamento com HBPM da TEP maciça com instabilidade hemodinâmi$\mathrm{ca}^{(30,31)}$. Considera-se TEP maciça quando a obstrução da circulação pulmonar envolve território de duas ou mais artérias lobares ou quantidade equivalente de êmbolos em outros vasos ${ }^{(7)}$.

2) Em pacientes na UTI e que necessitam freqüentemente de procedimento invasivos, a meia-vida longa da HBPM é inadequada(32).

3) Em portadores de insuficiência renal, devido à dificuldade de monitorização(14).

4) Em pacientes com significativo edema periférico que pode alterar a absorção da droga administrada por via subcutânea(32).
5) Nos casos de obesidade mórbida não existem estudos definindo a dosagem de HBPM ajustada para o peso corpóreo ${ }^{(8,9)}$.

\section{Conclusão}

Um grande número de estudos tem evidenciado que heparina de baixo peso molecular é tão segura e eficaz no tratamento da tromboembolia pulmonar quanto heparina não fracionada. Devido a suas características farmacocinéticas, podem ser usadas por via subcutânea uma ou duas vezes ao dia, sem necessidade de acesso venoso, bomba de infusão, e sem monitorização laboratorial freqüente.

Recentes publicações têm sugerido que o tratamento com HBPM em um grupo selecionado de pacientes com diagnóstico de TEP pode ser realizado exclusivamente no domicílio ou com período reduzido de hospitalização, desde que se disponha de mecanismos rigorosos de supervisão médica. Tal conduta pode conferir uma grande vantagem em termos de custos e satisfação do paciente.

\section{REFERÊNCIAS}

1. Moser KM. Venous thromboembolism. Am Rev Respir Dis 1990;141: 235-49.

2. Anderson FA, Wheller HB, Goldberg RJ, Hosmer DW, Patwardhan $N A$, J ovanovic $B$, et al. A population-based perspective of the hospital incidence and case-fatality rates of deep vein thrombosis and pulmonary embolism: the Worcester DVT study. Arch Intern Med 1991;151: 933-8.

3. Stein PD, Huang $H$, Afzal A, Noor HA. Incidence of acute pulmonary embolism in a general hospital. Chest 1999;116:909-13.

4. Dismuke SE, Wagner EH. Pulmonary embolism as a cause of death. J AMA 1986;255:2039-42.

5. Lindblad B, Sternby NH, Bergqvist D. Incidence of venous thromboembolism verified by necropsy over 30 years. Br Med J 1991;302:709. 11.

6. Maffei FHA, Faleiros ATS, Venezian CA, Franco MF. Contribuição ao estudo da incidência e anatomia patológica do tromboembolismo pulmonar em autópsias. Rev Assoc Med Bras 1980;26:7-9.

7. Menna Barreto S, Cerski MR, Gazzana MB, Stefani SD, Rossi R. Tromboembolia pulmonar em necropsias no Hospital de Clínicas de Porto Alegre, 1985-1995. J Pneumol 1997;23:131-6.

8. Hirsh J, Warkentin TE, Shaughnessy SG, Anand SS, Halperin J L, Raschke R, et al. Heparin and low-molecular-weight heparin. Chest 2001;119:64-94S.

9. Weitz JI. Low-molecular-weight heparins. N Engl J Med 1997;337: 688-98.

10. Pineo FG, Hull RD. Unfractioned and low-molecular-weight heparin. Med Clin North Am 1998;82:587-99.

11. Cruvinel MC, Morrone N. Heparinas de baixo peso molecular. J Pneumol 1995;21:185-8.

12. Hirsh J, Warkentin TE, Raschke R, Granger O, Ohman EM, Dalen JE, et al. Heparin and low-molecular-weight heparin. Chest 1998;114: 489-510S

13. Warkentin TE, Levine MN, Hirsh J, Hersewood P, Roberts RS, Gent $M$, et al. Heparin-induced trombocytopenia in patients treated with 
low-molecular-weight heparin or unfractioned heparin. N Engl J Med 1995;332:1330-5.

14. Litin SC, Heit JA, Mees KA. Use of low-molecular-weight heparin in the treatment of venous thromboembolic disease: answers to frequently asked questions. Mayo Clin Proc 1998;73:545-51.

15. Raschke RA, Reilly BM, Guidry RJ , Fontana RJ , Srinivas S. The weightbased heparin dosing nomogram compared with a standard care nomogram: a randomized controlled trial. Ann Intern Med 1993;119: 874-81.

16. Harrison L, J ohnston M, Massicote MP, Crowther M, Maffat $K$, Hirsh J, et al. Comparison of $5 \mathrm{mg}$ and $10 \mathrm{mg}$ loading doses in initiation of warfarin therapy. Ann Intern Med 1997;126:133-6.

17. Hyers TM, Agnelli C, Hull RD, Weg J G, Morris TA, Samama M, et al. Antithrombotic therapy for venous thromboembolic disease. Chest 1998;114:561-78S.

18. Albada J, Niewenhuis HK, Sixma JJ. Treatment of acute venous thromboembolism with low-molecular-weight heparin (Fragmin). Circulation 1989;80:935-40.

19. Thery C, Simonneau G, Meyer G, Helenon O, Bridey F, Armagnac C, et al. Randomized trial of sub cutaneous low-molecular-weight heparin (Fraxiparin) compared with intravenous unfractioned heparin in the curative treatment of submassive pulmonary embolism. Circulation 1992;85:1380-9.

20. The COLUMBUS Investigators. Low-molecular-weight heparin in the treatment of patients with venous thromboembolism. N Engl J Med 1997;337:657-62.

21. Simonneau G, Sors H, Charbonnier B, Page $Y$, Laaban JP, Azarian R, et al. A comparison of low-molecular-weight heparin with unfractioned heparin for acute pulmonary embolism. N Engl J Med 1997;337:6639.

22. Dolovich LR, Ginsberg JS, Douketis JD, Halbrock AM, Cheah G, et al. A meta-analysis comparing low-molecular-weight heparins with unfractioned heparin in the treatment of venous thromboembolism. Arch Intern Med 2000;160:181-8.
23. Prandoni P. Antithrombotic strategies in patients with cancer. Thromb Haemost 1997;78:141-4.

24. Hull RD, Raskob GE, Brant RF, Pineo GF, Elliot G, Stein P, et al. Lowmolecular-weight heparin vs heparin in the treatment of patients with pulmonary embolism. Arch Intern Med 2000;160:229-36.

25. Merli G, Spiro TE, Olson CG, Ulrich A, Davidson BL, Eldor A, et al. Subcutaneous Enoxaparin once or twice daily compared with unfractioned heparin for treatment of venous thromboembolic disease. Ann Intern Med 2001;134:191-202.

26. Levine $M$, J ent $M, H$ irsh J, Leclerc J, Anderson D, Weitz J, et al. A comparison of low-molecular-weight heparin administered primarily at home with unfractioned heparin administered in the hospital for proximal deep vein thrombosis. N Engl J Med 1996;334:677-81.

27. Koopman MMW, Prandoni P, Piovella F, Ockelfold PA, Bradjes PM, van Der Merr J, et al. Treatment of venous thrombosis with intravenous unfractioned heparin administered in the hospital as compared with subcutaneous low-molecular-weight heparin administered at home. N Engl J Med 1996;334:682-7.

28. Wells PS, Kovacs MJ, Bormanis J, Forgie MA, Goudie D, Morrow B, et al. Expanding eligibility for outpatient treatment of deep venous thrombosis and pulmonary embolism with low-molecular-weight heparin. Arch Intern Med 1998;158:1809-12.

29. Kovacs MJ, Anderson D, Morrow B, Gray L, Touchie D, Wells PS. Outpatient treatment of pulmonary embolism with Dalteparin. Thromb Haemost 2000;83:209-11.

30. Hyers TM, Agnelli G, Hull RD. Antithrombotic therapy for venous thromboembolic disease. Chest 2001;119:176-93S.

31. Hamel E, Pacouret G, Vincentelli D. Thrombolysis or heparin therapy in massive pulmonary embolism with right ventricular dilation. Chest 2001;120:120-5.

32. Legere BM, Dweik RA, Arroliga AC. Venous thromboembolism in the intensive care unit. Clin Chest Med 1999;20:367-84. 